\title{
ON CERTAIN POSITIVE SEMIDEFINITE MATRICES OF SPECIAL FUNCTIONS
}

\author{
RUIMING ZHANG
}

\begin{abstract}
Special functions are often defined as a Fourier or Laplace transform of a positive measure, and the positivity of the measure manifests as positive definiteness of certain matrices. The purpose of this expository note is to give a sample of such positive definite matrices to demonstrate this connection for some well-known special functions such as Gamma, Beta, hypergeometric, theta, elliptic, zeta and basic hypergeometric functions.
\end{abstract}

\section{INTRODUCTION}

Recall that for $n \in \mathbb{N}$ and $A=\left(a_{j, k}\right)_{j, k=1}^{n}, a_{j, k} \in \mathbb{C}, A$ is called positive semidefinite if and only if the quadratic form $\sum_{j, k=1}^{n} a_{j, k} z_{j} \overline{z_{k}} \geq 0$ for all $z_{1}, z_{2}, \ldots z_{n} \in$ $\mathbb{C}$, and it is positive definite if it is positive semidefinite and $\sum_{j, k=1}^{n} a_{j, k} z_{j} \overline{z_{k}}=0$ implies that $z_{1}=\cdots=z_{n}=0$. Given two positive semidefinite $n \times n$ matrices

$$
A=\left(a_{j, k}\right)_{j, k=1}^{n}, \quad B=\left(b_{j, k}\right)_{j, k=1}^{n}, \quad a_{j, k}, b_{j, k} \in \mathbb{C},
$$

it is well-known that the Schur (Hadamard) product $A \circ B=\left(a_{j, k} b_{j, k}\right)_{j, k=1}^{n}$ is also positive semidefinite, and it satisfies [4]

$$
\operatorname{det}(A \circ B) \geq \operatorname{det}(A) \cdot \operatorname{det}(B) .
$$

Since all the minors of a positive semidefinite matrix are nonnegative, hence a positive semidefinite matrix of special function entries can yield many inequalities for special functions.

Given a positive measure $\mu(x)$ on the real line $\mathbb{R}$, We denote $\mathcal{H}$ as the Hilbert space of $\mu$-square integrable functions,

$$
\mathcal{H}=\left\{\left.f\left|\int_{\mathbb{R}}\right| f(x)\right|^{2} \mu(d x)<\infty\right\}
$$

endowed with the usual inner product,

$$
<f, g>=\int_{\mathbb{R}} f(x) \overline{g(x)} \mu(d x), \quad f, g \in \mathcal{H} .
$$

Key words and phrases. Special functions; positive semidefinite matrices; special function inequalities.

This work is partially supported by National Natural Science Foundation of China, grant No. 11371294 and Northwest A\&F University of China. 
Let $\left\{f_{n}(x)\right\}_{n=0}^{N} \subset \mathcal{H}$, where $N$ may be any nonnegative integer in $\mathbb{N}_{0}$ or equals to $\infty$, then the finite sections of the Gram matrices [1, 5]

$$
G_{n}=\left(<f_{j}, f_{k}>\right)_{j, k=0}^{n}, \quad n=0, \ldots, N
$$

are positive semidefinite, and they are positive definite if $\left\{f_{n}(x)\right\}_{n=0}^{N} \subset \mathcal{H}$ are linearly independent.

In this article we shall list some of positive semidefinite matrices with special function entries. Our method to obtain positive semidefinite matrices is first to isolate an inner product structure associated with the special function, then choose a function set to compute the corresponding Gram matrices, and finally apply Schur product to the obtained more general positive semidefinite matrices. Even though the proofs are completely trivial, these positive semidefinite matrices sometimes may turn out to be very handy. In the following discussion if any of the formulas below are not specifically referenced, it means that they can be found in [2, 6]

\section{MAin Results}

Recall that the Jacobi $\theta_{3}$-function is defined by

$$
\theta_{3}(z, q)=\sum_{n=-\infty}^{\infty} q^{n^{2}} e^{2 \pi i n v}, \quad z=e^{2 \pi i v},|q|<1
$$

For $0<q<1$, define

$$
\mu(x)=\sum_{n=-\infty}^{\infty} q^{n^{2}} \delta(x-n)
$$

then $\mu(x)$ is a positive measure on $(-\infty, \infty)$. For $n \in \mathbb{N}, c_{1}, c_{2}, \ldots, c_{n} \in \mathbb{C}$ and $v_{j} \in \mathbb{C}, j=1, \ldots, n$ we have

$$
\begin{aligned}
& \int_{-\infty}^{\infty}\left|\sum_{j=1}^{n} c_{j} e^{2 \pi i v_{j} x}\right|^{2} d \mu(x)=\sum_{j, k=0}^{n} c_{j} \overline{c_{k}} \int_{-\infty}^{\infty} e^{2 \pi i x\left(v_{j}-\overline{v_{k}}\right)} d \mu(x) \\
& =\sum_{j, k=0}^{n} c_{j} \overline{c_{k}} \sum_{\ell=-\infty}^{\infty} q^{\ell^{2}} e^{2 \pi i \ell\left(v_{j}-\overline{v_{k}}\right)}=\sum_{j, k=0}^{n} c_{j} \overline{c_{k}} \theta_{3}\left(e^{2 \pi i\left(v_{j}-\overline{v_{k}}\right)}, q\right) \geq 0 .
\end{aligned}
$$

Thus, for $n \in \mathbb{N}, 0<q<1$ and $v_{j} \in \mathbb{C}, j=1, \ldots, n$, the matrix

$$
\left(\theta_{3}\left(e^{2 \pi i\left(v_{j}-\overline{v_{k}}\right)}, q\right)\right)_{j, k=1}^{n}
$$

is positive semi-definite. For

$$
v_{j, \ell} \in \mathbb{C}, 0<q_{\ell}<1,1 \leq j \leq n, 1 \leq \ell \leq m, \quad m, n \in \mathbb{N},
$$

by taking Schur product of the above matrix we prove that

$$
\left(\prod_{\ell=1}^{m} \theta_{3}\left(e^{2 \pi i\left(v_{j, \ell}-\overline{v_{k, \ell}}\right)}, q_{\ell}\right)\right)_{j, k=1}^{n}
$$

is also positive semidefinite.

The Jacobi elliptic function $\operatorname{dn}(2 K v)$ is defined by

$$
\operatorname{dn}(2 K v)=\frac{\pi}{K} \sum_{n \in \mathbb{Z}} \frac{q^{n}}{1+q^{2 n}} e^{2 n \pi v i}
$$


where

$$
|q|<1, K=\frac{\pi}{2} \theta_{3}^{2}(0, q), q e^{2 \pi|\Im(v)|}<1 .
$$

For $0<q<1$, let

$$
\mu(x)=\frac{\pi}{K} \sum_{n \in \mathbb{Z}} \frac{q^{n} \delta(x-n)}{1+q^{2 n}},
$$

is a positive measure on $\mathbb{R}$. For $n \in \mathbb{N}, c_{1}, c_{2}, \ldots, c_{n} \in \mathbb{C}$ and

$$
q e^{4 \pi\left|\Im\left(v_{j}\right)\right|}<1, v_{j} \in \mathbb{C}, j=1, \ldots, n,
$$

then the quadratic form

$$
\int_{-\infty}^{\infty}\left|\sum_{j=1}^{n} c_{j} e^{2 \pi i v_{j} x}\right|^{2} d \mu(x)=\sum_{j, k=1}^{n} c_{j} \overline{c_{k}} \operatorname{dn}\left(2 K\left(v_{j}-\overline{v_{k}}\right)\right)
$$

is nonnegative. Hence, for

$$
0<q<1, K=\frac{\pi}{2} \theta_{3}^{2}(0, q), q e^{4 \pi\left|\Im\left(v_{j}\right)\right|}<1,
$$

the matrix

$$
\left(\operatorname{dn}\left(2 K\left(v_{j}-\overline{v_{k}}\right)\right)\right)_{j, k=1}^{n}
$$

is positive semidefinite. By taking Schur product we see that

$$
\left(\prod_{\ell=1}^{m} \operatorname{dn}\left(2 K_{\ell}\left(v_{j, \ell}-\overline{v_{k, \ell}}\right)\right)\right)_{j, k=1}^{n}, \quad m, n \in \mathbb{N},
$$

is also positive semidefinite where

$$
0<q_{j}<1, K_{j}=\frac{\pi}{2} \theta_{3}^{2}\left(0, q_{j}\right), q_{j} e^{4 \pi\left|\Im\left(v_{j, \ell}\right)\right|}<1,1 \leq j \leq m .
$$

The Riemann zeta function is defined as the analytic continuation of the Dirichlet series [2, 6]

$$
\zeta(s)=\sum_{n=1}^{\infty} \frac{1}{n^{s}}, \quad \Re(s)>1 .
$$

For $\Re(s)>0$, it has the following integral representations,

$$
\frac{1}{s-1}-\frac{\zeta(s)}{s}=\int_{1}^{\infty} u^{-s} \frac{\{u\}}{u} d x, \quad \Re(s)>0,
$$

where $0 \leq\{x\}=x-\lfloor x\rfloor<1$ is the fractional part of $x$.

Given $n \in \mathbb{N}$, for $c_{j}, s_{j} \in \mathbb{C}, \Re\left(s_{j}\right)>0, j=1, \ldots, n$, we have

$$
\int_{1}^{\infty}\left|\sum_{j=1}^{n} \frac{c_{j}}{u^{s_{j}}}\right|^{2} \frac{\{u\}}{u} d x \geq 0 .
$$

Then for $n \in \mathbb{N}$, the matrix

$$
\left(\frac{1}{s_{j}+\overline{s_{k}}-1}-\frac{\zeta\left(s_{j}+\overline{s_{k}}\right)}{s_{j}+\overline{s_{k}}}\right)_{j, k=1}^{n}
$$

is positive semidefinite where $\Re\left(s_{j}\right)>0, j=1, \ldots, n$. 
For $m, n \in \mathbb{N}$, by taking Schur product we see the matrix

$$
\left(\prod_{\ell=1}^{m}\left\{\frac{1}{s_{j, \ell}+\overline{s_{k, \ell}}-1}-\frac{\zeta\left(s_{j, \ell}+\overline{s_{k, \ell}}\right)}{s_{j, \ell}+\overline{s_{k, \ell}}}\right\}\right)_{j, k=1}^{n}
$$

is also positive semidefinite where $\Re\left(s_{j, \ell}\right)>0, j=1, \ldots, n, \ell=1, \ldots, m$.

Recall the Euler Gamma function $\Gamma(z)$ is defined as the analytic continuation of integral,

$$
\Gamma(z)=\int_{0}^{\infty} e^{-x} x^{z-1} d x, \quad \Re(z)>0 .
$$

Then for $m, n \in \mathbb{N}$, by taking

$$
f_{k}(x)=x^{z_{k}}, d \mu(x)=e^{-x} \frac{d x}{x} 1_{\{x>0\}},
$$

then $<f_{j}, f_{k}>=\Gamma\left(z_{j}+\overline{z_{k}}\right)$, hence matrices

$$
\left(\Gamma\left(z_{j}+\overline{z_{k}}\right)\right)_{j, k=1}^{n}
$$

and

$$
\left(\prod_{\ell=1}^{m} \Gamma\left(z_{j, \ell}+\overline{z_{k, \ell}}\right)\right)_{j, k=1}^{n}
$$

are positive semidefinite for $\Re\left(z_{j}\right), \Re\left(z_{j, \ell}\right)>0,1 \leq j \leq n, 1 \leq \ell \leq m$.

For $\lambda>0$ and $0<\phi<\pi$ we have the following integral

$$
\frac{1}{2 \pi} \int_{-\infty}^{\infty} e^{(2 \phi-\pi) x}|\Gamma(\lambda+i x)|^{2} d x=\frac{\Gamma(2 \lambda)}{(2 \sin \phi)^{2 \lambda}},
$$

which is integral of the weight function for the Meixner-Pollaczek orthogonal polynomials. By taking function sequence $f_{j}(x)=e^{2 \phi_{j} x}, j=1, \ldots, n$ and $d \mu(x)=$ $e^{-\pi x}|\Gamma(\lambda+i x)|^{2} d x$ we see the matrix

$$
\left(\frac{1}{\sin ^{\lambda}\left(\phi_{j}+\phi_{k}\right)}\right)_{j, k=1}^{n}
$$

is positive semidefinite where $\lambda>0, \pi / 2>\phi_{j}>0, \quad j=1, \ldots, n, \quad n \in \mathbb{N}$. By taking the Schur product, the matrix

$$
\left(\frac{1}{\prod_{\ell=1}^{m} \sin ^{\lambda_{\ell}}\left(\phi_{j, \ell}+\phi_{k, \ell}\right)}\right)_{j, k=1}^{n}
$$

is also positive semidefinite where

$$
\frac{\pi}{2}>\phi_{j, \ell}>0, \lambda_{j}>0, \quad 1 \leq j \leq n, 1 \leq \ell \leq m, \quad m, n \in \mathbb{N} .
$$

Similarly, from the Euler's Beta function

$$
B(p, q)=\int_{0}^{1} x^{p}(1-x)^{q} \frac{d x}{x(1-x)}, \quad \Re(p), \Re(q)>0,
$$

we get that for $m, n \in \mathbb{N}$ the matrices, by considering

$$
f_{j}(x)=x^{p_{j}}(1-x)^{q_{j}} d \mu(x)=\frac{d x}{x(1-x)} 1_{\{0<x<1\}},
$$


we see the matrices

$$
\left(B\left(p_{j}+\overline{p_{k}}, q_{j}+\overline{q_{k}}\right)\right)_{j, k=1}^{n}
$$

and

$$
\left(\prod_{\ell=1}^{m} B\left(p_{j, \ell}+\overline{p_{k, \ell}}, q_{j, \ell}+\overline{q_{k, \ell}}\right)\right)_{j, k=1}^{n},
$$

are positive semidefinite where $\Re\left(p_{j}\right), \Re\left(p_{j, \ell}\right), \Re\left(q_{j}\right), \Re\left(q_{j, \ell}\right)>0,1 \leq j \leq n, 1 \leq$ $\ell \leq m$.

The shifted factorial is defined by [1, 3, 5]

$$
(a)_{n}=\frac{\Gamma(a+n)}{\Gamma(a)},\left(a_{1}, \ldots, a_{m}\right)_{n}=\prod_{k=1}^{m}\left(a_{k}\right)_{n},
$$

where $a, n, a_{1}, \ldots, a_{m} \in \mathbb{C}$. Then for $s+1 \geq r$ and $a_{1}, \ldots, a_{r}, b_{1}, \ldots, b_{s} \in \mathbb{C}$, the hypergeometric series is defined by

$$
{ }_{r} F_{s}\left(\begin{array}{c}
a_{1}, \ldots, a_{r} \\
b_{1}, \ldots, b_{s}
\end{array} \mid z\right)=\sum_{n=0}^{\infty} \frac{\left(a_{1}, \ldots, a_{r}\right)_{n}}{\left(1, b_{1}, \ldots, b_{s}\right)_{n}} z^{n}
$$

where $z \in \mathbb{C}$ for $s+1>r$ and $|z|<1$ for $s+1=r$. Given $m, n \in \mathbb{N}$ and nonnegative integers $r_{\ell}, s_{\ell}, 1 \leq \ell \leq m$, we assume that $a_{1, \ell}, \ldots, a_{r_{\ell}, \ell}, b_{1, \ell}, \ldots, b_{s_{\ell}, \ell}, 1 \leq \ell \leq m$ are so chosen such that

$$
\frac{\left(a_{1, \ell}, \ldots, a_{r_{\ell}, \ell}\right)_{n}}{\left(b_{1, \ell}, \ldots, b_{s_{\ell}, \ell}\right)_{n}} \geq 0, \quad 1 \leq \ell \leq m, n \in \mathbb{N}_{0} .
$$

Then the matrix

$$
\left(\prod_{\ell=1}^{m} r_{\ell} F_{s_{\ell}}\left(\begin{array}{c}
a_{1, \ell}, \ldots, a_{r_{\ell}, \ell} \\
b_{1, \ell}, \ldots, b_{s_{\ell}, \ell}
\end{array} \mid z_{j, \ell} \overline{z_{k, \ell}}\right)\right)_{j, k=1}^{n},
$$

is positive semidefinite where for $1 \leq \ell \leq m$ we assume that $z_{j, \ell} \in \mathbb{C}, 1 \leq j \leq n$ if $s_{\ell}+1>r_{\ell}$, and $\left|z_{j, \ell}\right|<1,1 \leq j \leq n$ if $s_{\ell}+1=r_{\ell}$.

From

$$
\left(1-2^{1-s}\right) \Gamma(s) \zeta(s)=\int_{0}^{\infty} u^{s} \frac{d u}{u\left(e^{u}+1\right)}, \quad \Re(s)>0,
$$

we see the matrix

$$
\left(\prod_{\ell=1}^{m}\left(1-2^{1-s_{j, \ell}-\overline{s_{k, \ell}}}\right) \Gamma\left(s_{j, \ell}+\overline{s_{k, \ell}}\right) \zeta\left(s_{j, \ell}+\overline{s_{k, \ell}}\right)\right)_{j, k=1}^{n}
$$

is positive semidefinite where $n, m \in \mathbb{N}$ and $\Re\left(s_{j, \ell}\right)>0, j=1, \ldots n, \ell=1, \ldots, m$,

From

$$
\left(1-2^{1-s}\right) \Gamma(s+1) \zeta(s)=\int_{0}^{\infty} u^{s} \frac{e^{u} d u}{\left(e^{u}+1\right)^{2}}, \quad \Re(s)>0
$$

we see that

$$
\left(\prod_{\ell=1}^{m}\left(1-2^{1-s_{j, \ell}-\overline{s_{k, \ell}}}\right) \Gamma\left(s_{j, \ell}+\overline{s_{k, \ell}}+1\right) \zeta\left(s_{j, \ell}+\overline{s_{k, \ell}}\right)\right)_{j, k=1}^{n}
$$

is positive semidefinite where $\Re\left(s_{j, \ell}\right)>0, j=1, \ldots n, \ell=1, \ldots, m, n, m \in \mathbb{N}$. 
Because

$$
\frac{\pi(s)_{p} \zeta(p+s)}{\sin \pi s}=\int_{0}^{\infty}\left((-1)^{p-1} \psi^{(p)}(1+x)\right) \frac{d x}{x^{s}}, \quad \Re(s) \in(0,1), p \in \mathbb{N},
$$

where

$$
\frac{(-1)^{p-1} \psi^{(p)}(1+x)}{p !}=\sum_{n=1}^{\infty} \frac{1}{(x+n)^{p+1}}
$$

is positive on $(0, \infty)$. Hence the matrix

$$
\left(\prod_{\ell=1}^{m} \frac{\left(s_{j, \ell}+\overline{s_{k, \ell}}\right)_{p_{\ell}} \zeta\left(p_{\ell}+s_{j, \ell}+\overline{s_{k, \ell}}\right)}{\sin \pi\left(s_{j, \ell}+\overline{s_{k, \ell}}\right)}\right)_{j, k=1}^{n}
$$

is positive semidefinite where $n, m, p \in \mathbb{N}$ and $0<\Re\left(s_{j, \ell}\right)<\frac{1}{2}, p_{\ell} \in \mathbb{N}, j=$ $1, \ldots, n, \ell=1, \ldots, m$.

The Riemann Xi function

$$
\Xi(z)=-\frac{\left(1+4 z^{2}\right)}{8 \pi^{(1+2 i z) / 4}} \Gamma\left(\frac{1+2 i z}{4}\right) \zeta\left(\frac{1+2 i z}{2}\right)
$$

is an even entire function of genus 1 . It satisfies

$$
\Xi(z)=\int_{-\infty}^{\infty} e^{-i t z} \phi(t) d t
$$

Thus the matrix

$$
\left(\prod_{\ell=1}^{m} \Xi\left(z_{j, \ell}-\overline{z_{k, \ell}}\right)\right)_{j, k=1}^{n}
$$

is positive semidefinite where $n, m \in \mathbb{N}$ and $z_{j, \ell} \in \mathbb{C}, j=1, \ldots, n, \ell=1, \ldots, m$.

The Hurwitz zeta function $\zeta(s, a)$ is defined as the analytic continuation of

$$
\zeta(s, a)=\sum_{n=0}^{\infty} \frac{1}{(n+a)^{s}}, \quad \Re(s)>1,-a \notin \mathbb{N}_{0}
$$

For $\Re(s)>0, a>0$ and $m, n \in \mathbb{N}$, from

$$
\left(\frac{1}{a^{s}}+\frac{1}{(1+a)^{s}}+\frac{(1+a)^{1-s}}{s-1}-\zeta(s, a)\right)=s \int_{1}^{\infty} \frac{\{x\} d x}{(x+a)^{s+1}},
$$

we see that

$$
\left(\prod_{\ell=1}^{m}\left(\frac{a_{\ell}^{-s_{j, \ell}-\overline{s_{k, \ell}}}+\left(1+a_{\ell}\right)^{-s_{j, \ell}-\overline{s_{k, \ell}}}-\zeta\left(s_{j, \ell}+\overline{s_{k, \ell}}, a_{\ell}\right)}{s_{j, \ell}+\overline{s_{k, \ell}}}+\frac{\left(1+a_{\ell}\right)^{1-s_{j, \ell}-\overline{s_{k, \ell}}}\left(s_{j, \ell}+\overline{s_{k, \ell}}\right)^{-1}}{\left(s_{j, \ell}+\overline{s_{k, \ell}}-1\right)}\right)\right)_{j, k=1}^{n},
$$

is positive semidefinite for $\Re\left(s_{j, \ell}\right), a_{\ell}, 1 \leq j \leq n, 1 \leq \ell \leq m$.

Since for $\Re(s)>0$ and $a>0$ we have

$$
\frac{\Gamma(s)}{4^{s}}\left(\zeta\left(s, \frac{a+1}{4}\right)-\zeta\left(s, \frac{a+3}{4}\right)\right)=\int_{0}^{\infty} x^{s} \frac{d x}{2 x e^{a x} \cosh x} .
$$

Then, the matrix

$$
\left(\prod_{\ell=1}^{m} \Gamma\left(s_{j, \ell}+\overline{s_{k, \ell}}\right)\left(\zeta\left(s_{j, \ell}+\overline{s_{k, \ell}}, \frac{a_{\ell}+1}{4}\right)-\zeta\left(s_{j, \ell}+\overline{s_{k, \ell}}, \frac{a_{\ell}+3}{4}\right)\right)\right)_{j, k=1}^{n}
$$


is positive semidefinite for $\Re\left(s_{j, \ell}\right), a_{\ell}>0,1 \leq j \leq n, 1 \leq \ell \leq m$.

The Lerch's Transcendent $\Phi(z, s, a)$ is defined as the analytic continuation of

$$
\Phi(z, s, a)=\sum_{n=0}^{\infty} \frac{z^{n}}{(a+n)^{s}}, \quad-z \notin \mathbb{N}_{0},|z|<1 ; \Re(s)>1,|z|=1 .
$$

For $a>0, z<1$ and $\Re(s)>0$ it has the following integral representation,

$$
\Gamma(s) \Phi(z, s, a)=\int_{0}^{\infty} x^{s} \frac{d x}{x e^{a x}\left(1-z e^{-x}\right)} .
$$

Then for $m, n \in \mathbb{N}$, the matrix

$$
\left(\prod_{\ell=1}^{m} \Gamma\left(s_{j, \ell}+\overline{s_{k, \ell}}\right) \Phi\left(z_{\ell}, s_{j, \ell}+\overline{s_{k, \ell}}, a_{\ell}\right)\right)_{j, k=1}^{n}
$$

is positive semidefinite for $\Re\left(s_{j, \ell}\right), a_{\ell}>0, z_{\ell}<1,1 \leq j \leq n, 1 \leq \ell \leq m$.

For $q \in(0,1)$ and $m \in \mathbb{N}$, let [1, 3, [5]

$$
(z ; q)_{\infty}=\prod_{n=0}^{\infty}\left(1-z q^{n}\right),(z, q)_{n}=\frac{(z ; q)_{\infty}}{\left(z q^{n} ; q\right)_{\infty}}, \quad z, n \in \mathbb{C},
$$

and

$$
\left(z_{1}, z_{2}, \ldots, z_{m} ; q\right)_{n}=\prod_{j=1}^{m}\left(z_{j} ; q\right)_{n}, z_{j}, n \in \mathbb{C} .
$$

For $\alpha_{1}, \alpha_{2}, \alpha_{3}, \alpha_{4}>0$, a weaker form of the Askey-Wilson beta integral is

$$
\begin{aligned}
& \int_{0}^{\pi} \frac{(1-q)^{5}(q ; q)_{\infty}^{6}\left|\left(e^{2 i \theta} ; q\right)_{\infty}\right|^{2} d \theta}{\left|\left(q^{\alpha_{1}} e^{i \theta}, q^{\alpha_{2}} e^{i \theta}, q^{\alpha_{3}} e^{i \theta}, q^{\alpha_{4}} e^{i \theta} ; q\right)_{\infty}\right|^{2} 2 \pi} \\
& =\frac{(1-q)^{2\left(\alpha_{1}+\alpha_{2}+\alpha_{3}+\alpha_{4}\right)} \prod_{1 \leq j<k \leq 4} \Gamma_{q}\left(\alpha_{j}+\alpha_{k}\right)}{\Gamma_{q}\left(\alpha_{1}+\alpha_{2}+\alpha_{3}+\alpha_{4}\right)} .
\end{aligned}
$$

By taking the function sequence,

$$
u_{k}(\theta)=\frac{1}{\left|\left(q^{\alpha_{k, 1}} e^{i \theta}, q^{\alpha_{k, 2}} e^{i \theta} ; q\right)_{\infty}\right|^{2}},
$$

and the Schur product, we see the matrix

$$
\left(\prod_{\ell=1}^{m} \frac{\Gamma_{q}\left(\alpha_{j, 1, \ell}+\alpha_{k, 1, \ell}\right) \Gamma_{q}\left(\alpha_{j, 2, \ell}+\alpha_{k, 2, \ell}\right)}{\Gamma_{q}\left(\alpha_{j, 1, \ell}+\alpha_{j, 2, \ell}+\alpha_{k, 1, \ell}+\alpha_{k, 2, \ell}\right)}\right)_{j, k=1}^{n}
$$

is positive semidefinite for

$$
m, n \in \mathbb{N}, \alpha_{k, 1, \ell}, \alpha_{k, 2, \ell}>0, \quad 1 \leq k \leq n, 1 \leq \ell \leq m .
$$

For $r, s \in \mathbb{N}_{0}, 0<q<1$, and $a_{1}, \ldots, a_{r}, b_{1}, \ldots, b_{s} \in \mathbb{C}$, let

$$
\begin{aligned}
& { }_{r} A_{s}^{(\alpha)}\left(a_{1}, \ldots, a_{r} ; b_{1}, \ldots, b_{s} ; q ; z\right)={ }_{r} \phi_{s}\left(\begin{array}{c}
a_{1}, \ldots, a_{r} \\
b_{1}, \ldots, b_{s}
\end{array} \mid q, z\right) \\
& =\sum_{n=0}^{\infty} \frac{\left(a_{1}, \ldots, a_{r} ; q\right)_{n}}{\left(b_{1}, \ldots, b_{s} ; q\right)_{n}} q^{\alpha n^{2}} z^{n} .
\end{aligned}
$$


Then it is clear that for $0<q<1$ and $s+1 \geq r$ we have

$$
\begin{aligned}
& { }_{r} A_{s}^{((s+1-r) / 2)}\left(a_{1}, \ldots a_{r} ; q, b_{1}, \ldots, b_{s} ; q ;(-1 / \sqrt{q})^{s+1-r} z\right) \\
& { }^{2}{ }_{r} \phi_{s}\left(\begin{array}{c}
a_{1}, \ldots, a_{r} \\
b_{1}, \ldots, b_{s}
\end{array} \mid q, z\right)
\end{aligned}
$$

where the basic hypergeometric series ${ }_{r} \phi_{s}$ is defined by [1, 3, 5]

$$
{ }_{r} \phi_{s}\left(\begin{array}{c}
a_{1}, \ldots, a_{r} \\
b_{1}, \ldots, b_{s}
\end{array} \mid q, z\right)=\sum_{n=0}^{\infty} \frac{\left(a_{1}, \ldots, a_{r} ; q\right)_{n}}{\left(q, b_{1}, \ldots, b_{s} ; q\right)_{n}}\left(-q^{(n-1) / 2}\right)^{n(s+1-r)} z^{n} .
$$

For $n, m \in \mathbb{N}$ and $0<q_{\ell}<1,1 \leq \ell \leq m$, we assume that $z_{j, \ell} \in \mathbb{C}, 1 \leq j \leq n$ when $\alpha_{\ell}>0$, and when $\alpha_{\ell}=0$, we restrict $z_{j, \ell}, 1 \leq j \leq n$ inside an open disk with certain radius less than 1 to ensure the associated series converges. Furthermore, we also assume that

$$
\frac{\left(a_{1, \ell}, \ldots, a_{r_{\ell}, \ell} ; q_{\ell}\right)_{n}}{\left(b_{1, \ell}, \ldots, b_{s_{\ell}, \ell} ; q_{\ell}\right)_{n}} \geq 0, \quad n \in \mathbb{N}_{0}
$$

then the matrix

$$
\left(\prod_{\ell=1}^{m} r_{\ell} A_{s_{\ell}}^{\left(\alpha_{\ell}\right)}\left(\begin{array}{c}
a_{1, \ell}, \ldots, a_{r_{\ell}, \ell} \\
b_{1, \ell}, \ldots, b_{s_{\ell}, \ell}
\end{array} \mid q_{\ell}, z_{j, \ell} \overline{z_{k, \ell}}\right)\right)_{j, k=1}^{n}
$$

are positive semidefinite.

For 3 ]

let

$$
q=e^{2 \pi i \sigma}, p=e^{2 \pi i \tau}, \quad \Im(\sigma), \Im(\tau)>0,
$$

$$
\begin{gathered}
\theta(x ; p)=(x, p / x ; p)_{\infty}, \theta\left(x_{1}, \ldots, x_{m} ; p\right)=\prod_{k=1}^{m} \theta\left(x_{k} ; p\right), \quad m \in \mathbb{N}, \\
(a ; q, p)_{n}= \begin{cases}\prod_{k=0}^{n-1} \theta\left(a q^{k} ; p\right), & n \in \mathbb{N}, \\
1 & n=0 \\
1 / \prod_{k=0}^{-n-1} \theta\left(a q^{n+k} ; p\right), & -n \in \mathbb{N}\end{cases}
\end{gathered}
$$

and

$$
\left(a_{1}, a_{2}, \ldots, a_{m} ; q, p\right)_{n}=\prod_{k=1}^{m}\left(a_{k} ; q, p\right)_{n}, \quad m \in \mathbb{N} .
$$

For $r, s \in \mathbb{N}_{0}$, the modular series ${ }_{r} E_{s}$ and ${ }_{r} G_{s}$ are defined by [3]

$$
\begin{aligned}
& { }_{r} E_{s}\left(a_{1}, \ldots, a_{r} ; b_{1}, \ldots, b_{s} ; q, p ; A, z\right) \\
& ={ }_{r} E_{s}\left(\begin{array}{c}
a_{1}, \ldots, a_{r} \\
b_{1}, \ldots, b_{s}
\end{array} \mid q, p ; A, z\right)=\sum_{n=0}^{\infty} \frac{\left(a_{1}, a_{2}, \ldots, a_{r} ; q, p\right)_{n}}{\left(q, b_{1}, \ldots, a_{s} ; q, p\right)_{n}} A_{n} z^{n}
\end{aligned}
$$

and

$$
\begin{aligned}
& { }_{r} G_{s}\left(c_{1}, \ldots, c_{r} ; d_{1}, \ldots, d_{s} ; q, p ; B, z\right) \\
& ={ }_{r} G_{s}\left(\begin{array}{c}
c_{1}, \ldots, c_{r} \\
d_{1}, \ldots, d_{s}
\end{array} \mid q, p ; B, z\right)=\sum_{n=-\infty}^{\infty} \frac{\left(c_{1}, \ldots, c_{r} ; q, p\right)_{n}}{\left(d_{1}, \ldots, d_{s} ; q, p\right)_{n}} B_{n} z^{n},
\end{aligned}
$$

where the sequences $A_{n}$ and $B_{n}$ are so-chosen to guarantee the above series converge in their appropriate domains.

Given $m \in \mathbb{N}, 1 \leq \ell \leq m$ we let

$$
r_{\ell}, s_{\ell} \in \mathbb{N}_{0}, q_{\ell}=e^{-2 \pi \sigma_{\ell}}, p_{\ell}=e^{-2 \pi \tau_{\ell}}, \sigma_{\ell}, \tau_{\ell}>0,
$$


and $a_{1, \ell}, \ldots, a_{r_{\ell}, \ell} ; b_{1, \ell}, \ldots, b_{s_{\ell}, \ell} ; c_{1, \ell}, \ldots, c_{r_{\ell}, \ell} ; d_{1, \ell}, \ldots, d_{s_{\ell}, \ell}$ and sequences $\left\{A_{n, \ell}\right\}_{n=0}^{\infty}$ and $\left\{B_{n, \ell}\right\}_{n=0}^{\infty}$ to ensure that

$$
\begin{gathered}
\frac{\left(a_{1, \ell}, \ldots, a_{r_{\ell}, \ell} ; q_{\ell}, p_{\ell}\right)_{n}}{\left(q_{\ell}, b_{1, \ell}, \ldots, a_{s_{\ell}, \ell} ; q_{\ell}, p_{\ell}\right)_{n}} A_{n, \ell} \geq 0, \quad n \in \mathbb{N}_{0}, \\
\quad \frac{\left(c_{1, \ell}, \ldots, c_{r_{\ell}, \ell} ; q_{\ell}, p_{\ell}\right)_{n}}{\left(d_{1, \ell}, \ldots, d_{s_{\ell}, \ell} ; q_{\ell}, p_{\ell}\right)_{n}} B_{n, \ell} \geq 0, \quad n \in \mathbb{Z}
\end{gathered}
$$

and the series ${ }_{r_{\ell}} E_{s_{\ell}} r_{\ell} G_{s_{\ell}}$ are convergent on some symmetric open subsets $S_{\ell} \subset \mathbb{C}$ and $T_{\ell} \subset \mathbb{C}$ with respect to the complex conjugation, then the matrices

$$
\left(\prod_{\ell=1}^{m} r_{\ell} E_{s_{\ell}}\left(\begin{array}{c}
a_{1, \ell}, \ldots, a_{r_{\ell}, \ell} \\
b_{1, \ell}, \ldots, b_{s_{\ell}, \ell}
\end{array} \mid q_{\ell}, p_{\ell} ; A_{\ell}, z_{j, \ell} \overline{z_{k, \ell}}\right)\right)_{j, k=1}^{n}
$$

and

$$
\left(\prod_{\ell=1}^{m} r_{\ell} G_{s_{\ell}}\left(\begin{array}{c}
c_{1, \ell}, \ldots, c_{r_{\ell}, \ell} \\
d_{1, \ell}, \ldots, d_{s_{\ell}, \ell}
\end{array} \mid q_{\ell}, p_{\ell} ; B_{\ell}, w_{j, \ell} \overline{w_{k, \ell}}\right)\right)_{j, k=1}^{n}
$$

are positive semidefinite where $z_{j, \ell} \in S_{\ell}, 1 \leq j \leq n, 1 \leq \ell \leq m$ and $w_{j, \ell} \in T_{\ell}, 1 \leq$ $j \leq n, 1 \leq \ell \leq m$.

\section{REFERENCES}

[1] G. E. Andrews, R. A. Askey, and R. Roy, Special Functions, Cambridge University Press, Cambridge, 1999.

[2] A. Erdelyi, Higher Transcendental Functions, Vols. I, II, III, A. Erdelyi, ed., McGraw-Hill. Reprinted by Krieger Publishing Co., Malabar, FL, 1981.

[3] G. Gasper and M. Rahman, Basic Hypergeometric Series, second edition Cambridge University Press, Cambridge, 2004.

[4] R. A. Horn and C. R. Johnson, Matrix Analysis, Cambridge University Press, Cambridge 1992.

[5] M. E. H. Ismail, Classical and Quantum Orthogonal Polynomials in one Variable, paperback edition, Cambridge University Press, Cambridge, 2009.

[6] F. W. J. Olver, D. W. Lozier, R. F. Boisvert, and C. W. Clark, NIST Handbook of Mathematical Functions, Cambridge University Press, 2010.

College of Science, Northwest A\&F University, Yangling, Shannxi 712100, P. R. CHINA.

E-mail address: ruimingzhang@yahoo.com 